\title{
A computational investigation of the red and blue shifts in hydrogen bonded systems ${ }^{\dagger}$
}

\author{
MITRADIP DAS ${ }^{\mathrm{a}}$ and SWAPAN K GHOSH ${ }^{\mathrm{b}, \mathrm{c}, *}$ \\ ${ }^{a}$ School of Chemical Sciences, National Institute of Science Education and Research, Bhubaneswar, Khordha, \\ Odisha 752 050, India \\ ${ }^{\mathrm{b}}$ Chemistry Group, Bhabha Atomic Research Centre, Mumbai, Maharashtra 400 085, India \\ ${ }^{\mathrm{c}}$ UM-DAE-Centre for Excellence in Basic Sciences, Vidyanagari Campus, Kalina, Santacruz (East), Mumbai, \\ Maharashtra 400 098, India \\ E-mail: swapankghosh@gmail.com
}

MS received 31 January 2017; revised 18 April 2017; accepted 23 May 2017

\begin{abstract}
The present work reports results of computational investigations of hydrogen bonding, with regard to the most common red shift in the vibrational frequency, as well as the less common blue shift in several hydrogen bonded systems. A few new correlations of the frequency shifts with the calculated electrostatic parameters are proposed, thereby generating new insight into both types of the frequency shifts. Thus, the frequency shifts in $\mathrm{X}-\mathrm{H}-\mathrm{Y}$ hydrogen bonded systems at different $\mathrm{H}-\mathrm{Y}$ distances are shown to correlate well with the Mulliken charges on $\mathrm{H}$ and $\mathrm{Y}$, with the positive and negative charges on $\mathrm{Y}$ correlating with the blue and red shift of the frequency of $\mathrm{X}-\mathrm{H}$ vibration, respectively. The role played by charge transfers at other parts of the interacting system is also discussed.
\end{abstract}

Keywords. Hydrogen bonding; blue shift; red shift; mulliken atomic charge.

\section{Introduction}

Hydrogen bonding ${ }^{1-3}$ is one of the most widely studied non-covalent interactions till date. It has been defined by Arunan et al., ${ }^{2}$ as, "an attractive interaction between a hydrogen atom from a molecule or a molecular fragment $X-H . . . Y$ in which $X$ is more electronegative than $H$, and an atom or a group of atoms in same or a different molecule, in which there is evidence of bond formation". Hydrogen bond is usually thought to lengthen the $\mathrm{X}-\mathrm{H}$ bond of an $\mathrm{X}-\mathrm{H}$...Y system, and hence would be associated with red shift in the corresponding vibrational frequency. However, there are enough theoretical and experimental evidences for $\mathrm{X}-\mathrm{H}$ bond shortening in some cases, and hence blue shift is observed in the stretching frequency. These hydrogen bonds have been termed as "improper hydrogen bonds" by Hobza and Havlas. $^{4}$

The lengthening of the $\mathrm{X}-\mathrm{H}$ bond has been understood as "a consequence of a stabilizing interaction". 5 In terms of electrostatics, the electron rich $\mathrm{Y}$ pulls the $\mathrm{H}$ towards itself, causing an elongation of the $\mathrm{X}-\mathrm{H}$ bond,

\footnotetext{
*For correspondence

${ }^{\dagger}$ Dedicated to the memory of the late Professor Charusita Chakravarty.
}

and hence a red shift. In terms of hyperconjugation, the red shifted hydrogen bond can be well understood as a donation or overlap of electron of the $\pi$ system or lone pair in $\mathrm{Y}$ to the $\sigma^{*}$ orbital of the $\mathrm{X}-\mathrm{H}$ bond. This transfer of electron to the anti-bonding orbital causes a lengthening and hence weakening of the $\mathrm{X}-\mathrm{H}$ bond, resulting into a red-shift in the $\mathrm{X}-\mathrm{H}$ infrared stretching frequency. However, it seems that blue-shifted hydrogen bonds fail to conform to any of these explanations. Different authors have thus proposed different theories for this, and some are discussed here.

Li et al., ${ }^{6}$ in 2002, analysed different possible criteria for the formation of blue shifted hydrogen bond, all of which have been examined later. They discussed the effect of groups attached to $\mathrm{X}$ in the bond forming molecule, the possibility of charge transfer and the orbital interaction of the molecules. In the first possibility, the relation between the interaction energy and gas phase basicity has been well-correlated, and the blue shift is claimed to occur at intermediate basicity. The second possibility has been taken up and led to the most acceptable theory and has been explained further by Joseph and Jemmis. ${ }^{5}$ The third possibility has been examined to explain the exceptions from the red shift, for example, by Alabugin et al., ${ }^{7}$ who considered the shift in hydrogen bond to be due to a balance 
between hyperconjugation and the s-character of the bond. The former tends to weaken the $\mathrm{X}-\mathrm{H}$ bond and an increase in the latter tends to strengthen the $\mathrm{X}-\mathrm{H}$ bond. The red or blue shift depends on the dominance of hyperconjugation or s-character respectively, which in turn is dependent on the polarization and the $\sigma$ acceptance of $\mathrm{X}-\mathrm{H}$. The concept of rehybridization has also been invoked and considered to be responsible for the improper blue shift in hydrogen bonding. They also concluded that $\mathrm{X}-\mathrm{H}$ bond will be shortened for systems like $\mathrm{F}_{3} \mathrm{SiH}$... $\mathrm{NH}_{3}$ due to lack of hyperconjugation. The possibility has been further examined by $\mathrm{Li}^{8}$ who brought in the concepts of hyperconjugation and rehybridization. The cause of electron transfer from the donor to the orbital of the $\mathrm{X}-\mathrm{H}$ (for $\mathrm{X}-\mathrm{H}$...Y system) is responsible for the lengthening of $\mathrm{X}-\mathrm{H}$ bond and has been explained by hyperconjugation. Rehybridization has been made responsible for shortening of the $\mathrm{X}-\mathrm{H}$ bond and hence an increase in the stretching frequency. Both red and blue shift observed in hydrogen bond can be considered as an effect of combination of these two factors.

Hobza ${ }^{9}$ introduced a new term "H-index", which is defined as the ratio of electron density transferred to the $\sigma$ orbital of $\mathrm{X}-\mathrm{H}$ and the total electron density in an $\mathrm{X}-\mathrm{H}$...Y system. There is a red shift observed when the value of $\mathrm{H}$-index becomes close to unity and a blue shift when it is less than 0.3. This implies that for red shift to occur, the total electron density transferred from the hydrogen bond acceptor moves to the orbital and hence the bond is weakened. This also implies that for blue shift, the electron density transfer from Y, if there is any at all, occurs mainly to the atomic orbital and not to the molecular orbital. He also reports about the negative values of the $\mathrm{H}$-index in some cases, which implies that there is electron density transfer from $\mathrm{H}$ to $\mathrm{Y}$, an evidence of "anti-hydrogen bonds". ${ }^{10}$

Joseph and Jemmis, ${ }^{5}$ based on their computational investigation, examined the situation and came up with an explanation that the $\mathrm{X}-\mathrm{H}$ bond energy, and hence its stretching infrared frequency, changes with change in $\mathrm{H}$... Y distance. According to them, on bringing $\mathrm{Y}$ closer to $\mathrm{H}$ in $\mathrm{X}-\mathrm{H}$... Y system, red shift is observed initially due to electrostatic interaction between the positive charge on $\mathrm{H}$ and negative charge on $\mathrm{Y}$, which elongates the $\mathrm{X}-\mathrm{H}$ bond. After a threshold distance, there is some electron density transfer from $\mathrm{Y}$ to $\mathrm{H}$ and so the $\mathrm{X}-\mathrm{H}$ bond starts being strengthened. This may result in blue shift of the $\mathrm{X}-\mathrm{H}$ stretching infrared frequency. On bringing $\mathrm{Y}$ further closer, after a certain threshold distance, there is a saturation of electron density on $\mathrm{H}$, and the $\mathrm{X}-\mathrm{H}$ bond starts becoming longer.
This can be also understood as filling up of electron density to the $\sigma^{*}$ orbital of the $\mathrm{X}-\mathrm{H}$ bond from $\mathrm{Y}$.

There have been many works on the issue of two types of frequency shifts reporting a large number of correlations of various quantities with the objective of rationalising or predicting whether there will be red or blue shift. However, the issue is still not resolved and there are many attempts to provide further insight through several calculations ${ }^{11-24}$ and experimental investigations. ${ }^{24-28}$

There is thus no single model or explanation that can definitely point out whether a hydrogen bond will show blue shift or red shift in its stable configuration. The main objective of this work is to explore a few new correlations for such a prediction by putting forward evidences from computational studies. The models and explanations have thus been proposed based on the present computational results and also based on the available current knowledge on this aspect. In what follows, we present computational details in Section 2, and discuss the calculated results along with the observed correlations in Section 3. Finally, we offer a few concluding remarks in section 4 .

\section{Computational details}

All the electronic structure calculations have been carried out by using the software GAMESS ${ }^{29}$ (General Atomic and Molecular Electronic Structure System). The fundamental methodology used here involves density functional theory (DFT) with the B3LYP exchange correlation functional (and also MP2 level) with 6-31G as the basis set with tight convergence criteria. The main calculations involve optimization of various hydrogen bonded systems by regulating the H...Y distance and studying the other characteristics including the total energy, $\mathrm{X}-\mathrm{H}$ bond length, infrared stretching frequency, $\mathrm{XY}$ distance, Mulliken charges on all X, H and Y, etc.

\section{Results and Discussion}

The hydrogen bonded systems considered here for electronic structure calculations cover the interaction of one of the species $\mathrm{CHF}_{3}, \mathrm{CHCl}_{3}, \mathrm{HCN}$ and $\mathrm{HF}$ with $\mathrm{NF}_{3}, \mathrm{NH}_{3}, \mathrm{NCl}_{3}, \mathrm{H}_{2} \mathrm{O}, \mathrm{HF}$, etc. Besides considering the optimized structure of the interacting systems for all the above systems, more detailed investigations have been carried out on two selected systems (a blue shifted system $\mathrm{F}_{3} \mathrm{CH} \ldots \mathrm{NF}_{3}$ and a red shifted system $\mathrm{F}_{3} \mathrm{CH} \ldots \mathrm{NH}_{3}$ ) by geometry optimization carried out at different fixed H. . . Y distances. In addition to the energy and structural parameters, several electrostatic parameters have also been calculated. 
The results obtained from the electronic structure calculations indicate the dependence of red or blue shift of hydrogen bonded systems on electrostatic paramaters, based on their Mulliken charges. From Table 1, it is apparent that the Mulliken charge on $\mathrm{H}$ is always positive, and blue shift seems to arise from the presence of positive Mulliken charge on $\mathrm{Y}$ in an $\mathrm{X}-\mathrm{H}$... Y system, while the red shift corresponds to negative charge on Y. Considering this fact intutively, if $\mathrm{X}$ and $\mathrm{Y}$ have their positions fixed, the negative Mulliken charge on Y pulls the $\mathrm{H}$ away from $\mathrm{X}$, which is covalently bonded with $H$. The resultant effect seems to lead to weakening of the $\mathrm{X}-\mathrm{H}$ bond and hence a red shift in the stretching frequency, as usually observed. On the other hand, a positive Mulliken charge on $\mathrm{Y}$ implies a repulsion between $\mathrm{H}$ and $\mathrm{Y}$, thus bringing $\mathrm{X}$ closer to $\mathrm{H}$ and hence introducing a blue shift in the stretching frequency of $\mathrm{X}-\mathrm{H}$.

An extension of this model can be considered by using the effective potential created on $\mathrm{X}-\mathrm{H}$ bond by $\mathrm{Y}$. A positive potential, which is a direct manifestation of the positive Mulliken charge on $\mathrm{Y}$, leads to a force on $\mathrm{H}$ towards $\mathrm{X}$, introducing a blue shift. The negative Mulliken charge on $\mathrm{Y}$, on the other hand, generates a negative potential on the $\mathrm{X}-\mathrm{H}$ bond, causing a red shift in its stretching frequency. This potential based approach has a direct implication for the systems having conjugated $\pi$-cloud of electrons, where the Mulliken charge cannot be defined directly. One of the direct consequences of this type of interaction is that the hydrogen bonds with benzene and hexaflourobenzene are blue shifted, which has both theoretical and experimental evidences.

From the results reported in Table 1, it is observed that the red shift occurs at lower values of $\mathrm{R}(\mathrm{HY})$ as compared to blue shift, which also seems to owe its origin to the electrostatic model. The attractive interaction between $\mathrm{H}$ and $\mathrm{Y}$ accounts for relatively lower values of $\mathrm{R}(\mathrm{HY})$ in case of red shift while the opposite is true for blue shift. To obtain further insight into these aspects, we report in Tables S1 and S2 (see Supplementary Information), the calculated results on various quantities of interest as a function of the R(HY) distance. Some of these results are shown as plots in Figures 1 to 5, for obtaining a pictorial view.

In Figure 1, we show the plot of total energy versus $\mathrm{H} . . . \mathrm{Y}$ distance for both blue $\left(\mathrm{F}_{3} \mathrm{CH} \ldots \mathrm{NF}_{3}\right)$ and red $\left(\mathrm{F}_{3} \mathrm{CH} . . . \mathrm{NH}_{3}\right)$ shifted systems, as calculated by using DFT with B3LYP exchange correlation. From the figure, it is observed that on moving $\mathrm{Y}$ further away from $\mathrm{H}$, the potential energy curve converges to zero at larger distance, from the positive side for the blue shifted system, which in turn suggests the effect of a repulsive force. The effect of an attractive central force is suggested by the fact that at any value of $\mathrm{R}(\mathrm{HY}), \Delta \mathrm{E}$ for

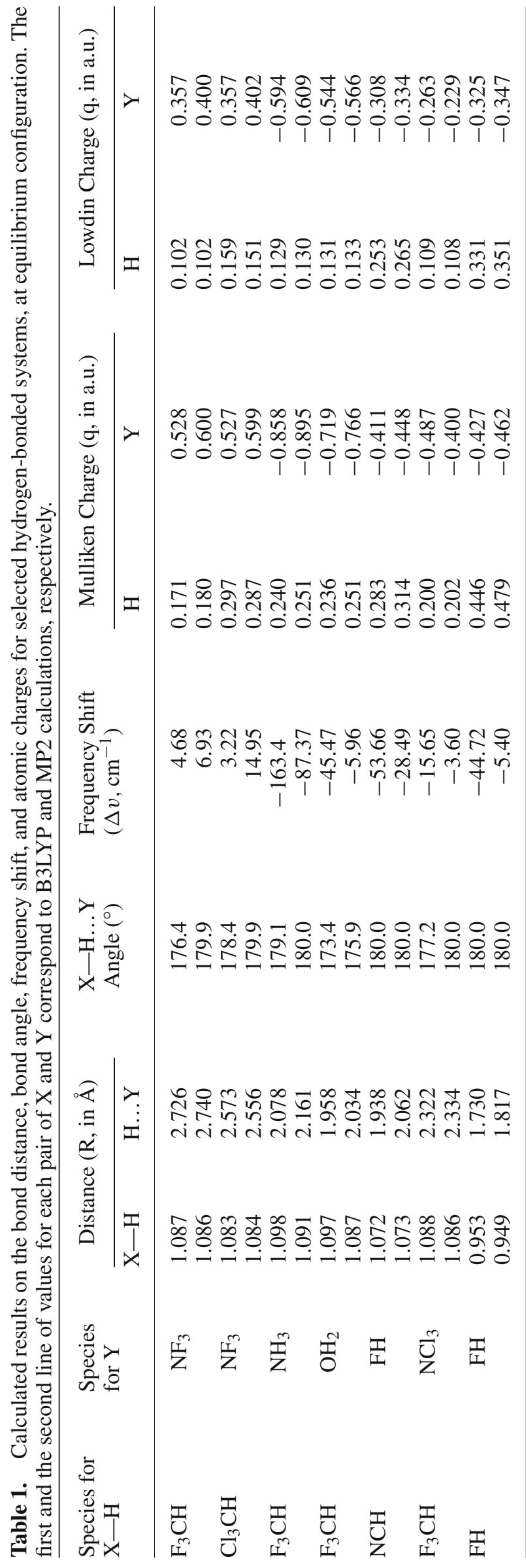




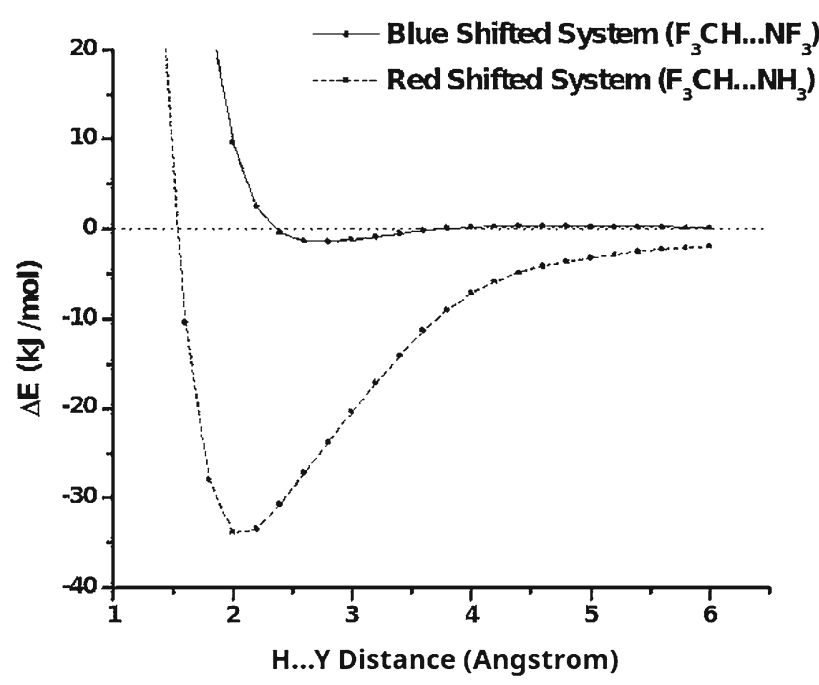

Figure 1. Plot of the Total Energy versus $\mathrm{H}$...Y distance for both blue $\left(\mathrm{F}_{3} \mathrm{CH} . . \mathrm{NF}_{3}\right)$ and red $\left(\mathrm{F}_{3} \mathrm{CH} . . . \mathrm{NH}_{3}\right)$ shifted systems, as calculated by using DFT with B3LYP exchange correlation.

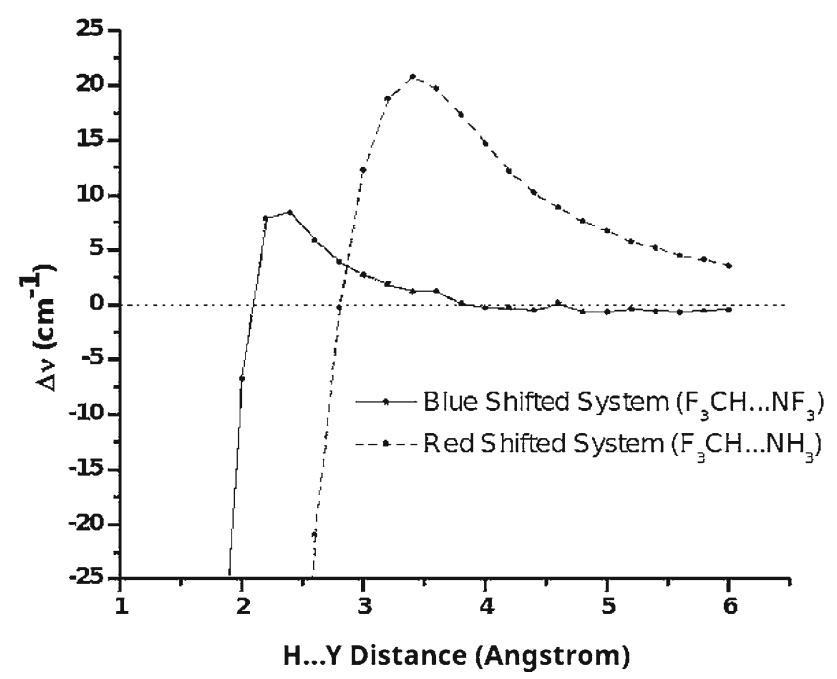

Figure 2. Plot of the shift in $\mathrm{X}-\mathrm{H}$ streching frequency versus $\mathrm{H}$...Y distance for both blue $\left(\mathrm{F}_{3} \mathrm{CH} . . . \mathrm{NF}_{3}\right)$ and red $\left(\mathrm{F}_{3} \mathrm{CH} . . . \mathrm{NH}_{3}\right)$ shifted systems, as calculated by using DFT with B3LYP exchange-correlation.

the red shifted case is lower than that of the blue shifted one. For a mathematical verification, the following equation has been considered for the potential energy curve, $\mathrm{U}(\mathrm{R})=\Delta \mathrm{E}(\mathrm{R})$, with the second term arising from electrostatics:

$$
U(R)=U_{0}(R)+\zeta \frac{q_{H} q_{Y}}{R}
$$

Considering $\zeta$ to be the same for both red and blue shifted systems for any value of R(HY), it is found that $U_{0}(R)$ showed a very good correlation with the Morse

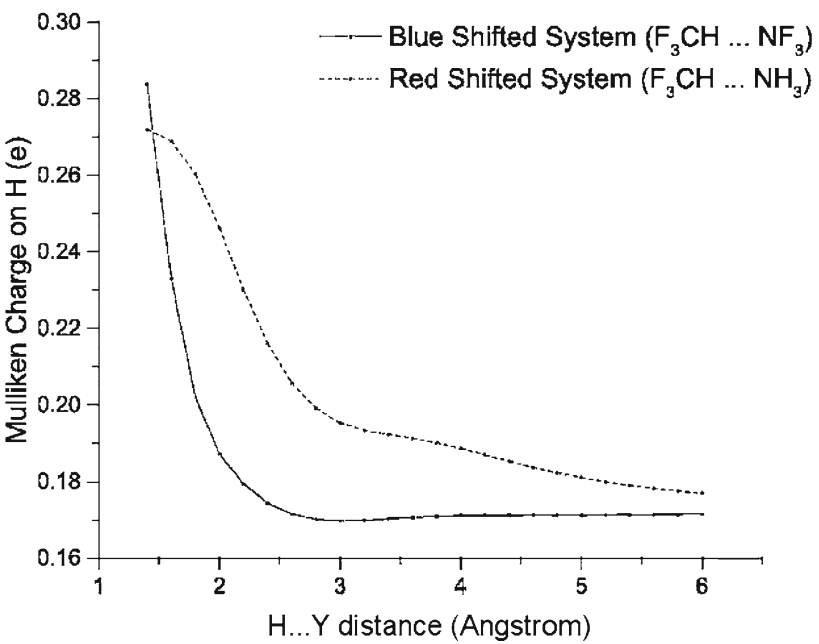

Figure 3. Plot of Mulliken Charge on $\mathrm{H}$ versus $\mathrm{H}$... distance for the blue $\left(\mathrm{F}_{3} \mathrm{CH} . . . \mathrm{NF}_{3}\right)$ and red $\left(\mathrm{F}_{3} \mathrm{CH} . . . \mathrm{NH}_{3}\right)$ shifted systems, as calculated by using DFT with B3LYP exchange correlation.

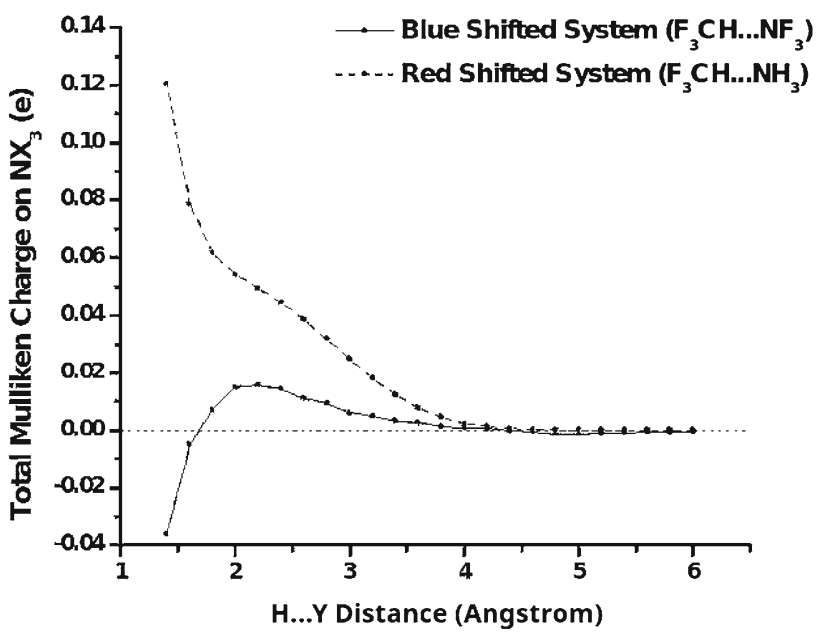

Figure 4. Plot of Total Mulliken Charge on $\mathrm{NX}_{3}$ versus $\mathrm{H}$...Y distance for both blue $\left(\mathrm{F}_{3} \mathrm{CH}\right.$...NF3) and red $\left(\mathrm{F}_{3} \mathrm{CH} . . . \mathrm{NH}_{3}\right)$ shifted systems, as calculated by using DFT with B3LYP exchange correlation.

potential. Using the standard equation for the Morse Potential, given by,

$$
U_{0}(R)=D_{e}\left[\left(1-\mathrm{e}^{-a\left(R-R_{0}\right)}\right)^{2}-1\right]
$$

it was found, by curve fitting, that the classical depth of the potential well, $D_{e}$ is $10.54 \mathrm{~kJ} / \mathrm{mole}$; the curvature related parameter, $a$, is $1.301 \AA^{-1}$ and the equilibrium distance $R_{0}$ is $2.455 \AA$, corresponding to a regression of 0.9999 for the fitting.

The total energy of hydrogen bonded systems does not owe its origin only to the electrostatic interaction, but also has other components including energy 

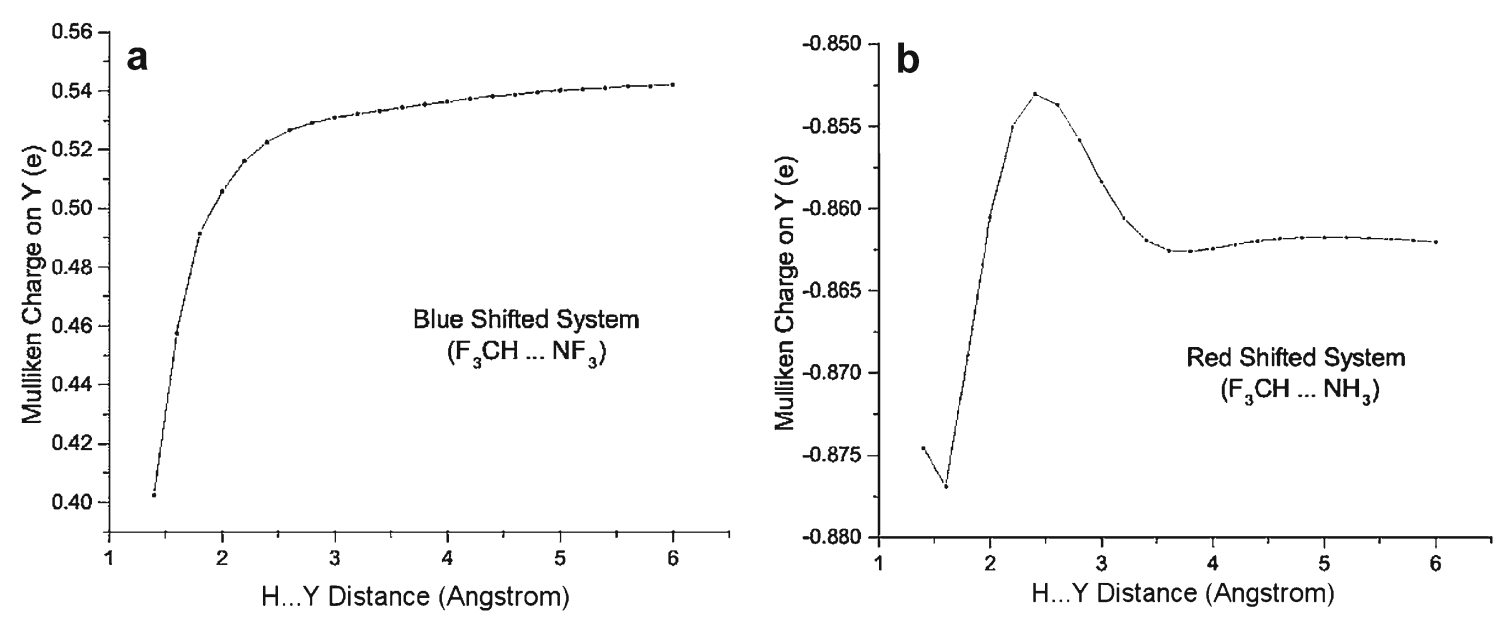

Figure 5. Plot of Mulliken charge on $\mathrm{Y}$ (here $\mathrm{N}$ ) versus $\mathrm{H} . . . \mathrm{Y}$ distance for both blue shifted $\left(\mathrm{F}_{3} \mathrm{CH}_{\mathrm{N}} . . \mathrm{NF}_{3}\right)$ (a) and red shifted $\left(\mathrm{F}_{3} \mathrm{CH} . . . \mathrm{NF}_{3}\right)(\mathbf{b})$ systems, as calculated by using DFT with B3LYP exchange correlation.

due to charge transfer, exchange energy and correlation energy. ${ }^{30,31}$ Although a shallow potential well for blue shifted hydrogen bonded systems show that electrostatic interactions play a major role, but there is still a bound state for the system. In fact, the decomposition of energy into electrostatic and non-electrostatic parts and correlation of the latter to Morse potential suggest the presence of energy component in the system which is not of electrostatic origin.

The next quantity of interest is the shift in $\mathrm{X}-\mathrm{H}$ stretching frequency as a function of the $\mathrm{H}$... Y distance for both the blue $\left(\mathrm{F}_{3} \mathrm{CH} . . . \mathrm{NF}_{3}\right)$ and red $\left(\mathrm{F}_{3} \mathrm{CH} \ldots \mathrm{NH}_{3}\right)$ shifted systems. A plot of the shift in the calculated $\mathrm{X}-\mathrm{H}$ stretching frequency versus $\mathrm{R}(\mathrm{HY})$, as shown in Figure 2, shows that the curves for blue and red shifted systems are qualitatively quite similar. On bringing $\mathrm{Y}$ closer to $\mathrm{H}$, an increase in shift is observed initially, which reaches a maximum and then decreases to give a red shift at lower values of R(HY). It is not difficult to understand that $\mathrm{R}(\mathrm{HY})$ should have lower values for red shifted system than the blue shifted system, the reasons for which has already been discussed. The maximum of the frequency suggests that at this distance the electrostatic effects become less pronounced and charge transfer effect dominates, as observed from the abrupt lowering of electron density in Figure 3. This correlation also complies with the theory proposed by Joseph and Jemmis. ${ }^{5}$ It may be noted that blue and red shifts are not specific to $\mathrm{H}$-bonding alone but are also observed in other cases, such as in the $\mathrm{CO}$ stretching frequency due the interaction of $\mathrm{CO}$ with suitable molecular systems. ${ }^{32}$

Since we discuss various aspects of the H-bond using electrostatics, it will be of interest to look at the partial atomic charges on the $\mathrm{H}$ atom participating in
H-bonding. In Figure 3, we show the variation of the calculated Mulliken charge on $\mathrm{H}$ with respect to change in $\mathrm{R}(\mathrm{HY})$. It is observed that on bringing $\mathrm{Y}$ closer to $\mathrm{H}$ from a larger distance, the charge on $\mathrm{H}$ for blue shifted system first decreases slowly up to a certain point and then increases. The decrease can be rationalized through electrostatics in which the positive charge on Y, in order to reduce the electrostatic repulsion energy, tends to push the positive charge away from $\mathrm{H}$ (essentially by attracting electrons to $\mathrm{H}$ ). The opposite is however true for the red shifted system, in which there is a decrease in electron density on $\mathrm{H}$ due to negative Mulliken charge on $\mathrm{Y}$, thus showing an increase in the charge on $\mathrm{H}$ throughout the range. On moving $\mathrm{Y}$ very close to $\mathrm{H}$, one observes an increase in the charge on $\mathrm{H}$, even for the blue shifted system, perhaps due to electron density transfer to the remote part of $\mathrm{F}_{3} \mathrm{CH}$, often assisted by the $\mathrm{NF}_{3}$ group (the so called group interaction). The rather slow variation in the charge on $\mathrm{H}$ at short $\mathrm{R}(\mathrm{HY})$ (in the range $\sim 1.4-1.8 \AA$ ) for red shifted system may be a consequence of electron density donation from $\mathrm{NH}_{3}$. More details on this aspect will be clearer from the consideration of the total charge on the $\mathrm{NX}_{3}$ as a whole, as discussed below.

In Figure 4, the calculated total charges on the $\mathrm{NF}_{3}$ and $\mathrm{NH}_{3}$ are plotted as a function of $\mathrm{R}(\mathrm{HY})$ to have a clear picture of the nature of charge transfer taking place. For $\mathrm{NH}_{3}$ (red-shifted system), the increase in the Mulliken charge on $\mathrm{NH}_{3}$ on bringing it closer to $\mathrm{H}$ suggests an electron density transfer from $\mathrm{NH}_{3}$ to $\mathrm{H}$, which in turn is a characteristic of the hydrogen bond. For $\mathrm{NF}_{3}$ (blue-shifted system), there is initially an increase in the Mulliken charge on bringing $\mathrm{H}$ and $\mathrm{NF}_{3}$ closer and then there is a decrease at a rather close distance. The 
decrease suggests an electron density transfer from $\mathrm{H}$ to $\mathrm{NF}_{3}$, which implies an anti-hydrogen bonding interaction.

While Figure 4 deals with the total charge on the $\mathrm{NX}_{3}$ species, more insight into the nature of the charge transfer can be obtained by looking at the charge on the $\mathrm{N}$ center of these species. Accordingly, in Figure 5, we plot the calculated Mulliken charges on $\mathrm{N}$ for both these two systems. For the blue shifted interaction, the charge on $\mathrm{N}$ is found to decrease monotonically on bringing the hydrogen bonding interaction participants closer (decrease in $\mathrm{R}(\mathrm{HY})$ ), which implies an electron density transfer to $\mathrm{N}$ from $\mathrm{H}$ and also eventually to $\mathrm{F}$. This can be understood as a consequence of a stabilizing interaction involving electrostatics, where the system attempts to minimize the repulsive energy between $\mathrm{N}$ and $\mathrm{H}$. However, for the red shifted system, there is electron density donation from the remote $\mathrm{H}$ to $\mathrm{N}$ and also from $\mathrm{N}$ to $\mathrm{H}$ of the hydrogen bonding interaction. The initial decrease, followed by an increase and an eventual decrease, leading to the consequent occurrence of the observed peak owe their origin to the unequal nature of the two electron density transfers and the dependence on the distance.

For a more detailed picture, the numerical values of various calculated quantities are shown in Tables $\mathrm{S} 1$ and S2, which also include the R(HY) dependence on a few other parameters as well, such as the $\mathrm{X}-\mathrm{H}$ bond length and bond order, the bond angle, etc. While the bond order variation is monotonic, the bond length variation shows slight non monotonic behavior. It may be noted that throughout the discussion on the various correlations presented here, we have focused on the electrostatic interactions between the two main participating $(\mathrm{H}$ and $\mathrm{Y})$ atoms, and the same between $\mathrm{X}$ and $\mathrm{H}$ or $\mathrm{X}$ and $\mathrm{Y}$ have not been accounted for. While at first sight this might appear as an apparent incompleteness, it is really not the case since the bonding interaction between $\mathrm{X}-\mathrm{H}$ is covalent with a clear dominance of quantum mechanical interaction over electrostatics. Also, as far as interaction between $\mathrm{X}$ and $\mathrm{Y}$ is concerned, it is the larger distance and the shielding due to electron density of the $\mathrm{X}-\mathrm{H}$ covalent bond, which play the role for minimized electrostatic effects in this case.

\section{Conclusions}

It can be concluded that the red or blue shift in $\mathrm{X}-\mathrm{H}$...Y hydrogen bonded systems depends on the Mulliken charge present on $\mathrm{H}$ and $\mathrm{Y}$. The positive Mulliken charge on $\mathrm{Y}$ is the cause of blue shift as it tends to shorten the $\mathrm{X}-\mathrm{H}$ bond length by electrostatic repulsion. The opposite is true for red shifted systems, where the negative charge on $\mathrm{Y}$ tends to lengthen the $\mathrm{X}-\mathrm{H}$ bond due to electrostatic attraction. This correlation can be extended to the conjugated $\pi$ bonded system by considering the electrostatic potential present at the centre of the benzene and fluorobenzene. The positive values of the potential implies a shortening of the $\mathrm{X}-\mathrm{H}$ bond length due to electrostatic repulsion of $\mathrm{H}$ towards $\mathrm{X}$. The plot for energy shows the presence of electrostatic component in it. It has also been confirmed by subtracting the electrostatic component from the total energy and the non-electrostatic contribution is observed to be close to the Morse potential. The Mulliken charges on $\mathrm{H}$ and $\mathrm{Y}$ show the transfer of electron density. In a nutshell, the shift in the $\mathrm{X}-\mathrm{H}$ stretching frequency in the hydrogen bonded system $\mathrm{X}-\mathrm{H} \ldots \mathrm{Y}$ can be attributed to the Mulliken charges present on $\mathrm{H}$ and $\mathrm{Y}$, and the associated electrostatic interaction.

\section{Supplementary Information (SI)}

Tables S1-S2 are given in the Supplementary Information which is available at www.ias.ac.in/chemsci.

\section{Acknowledgements}

We are thankful to all the three National Science Academies of India for the award of Academy Summer Research Fellowship (2016) and DST, India for the award of INSPIRE fellowship to one of us (MD). SKG thanks DAE, India for the Raja Ramanna Fellowship and UM-DAE-Centre of Excellence for Basic Sciences, Mumbai for a Distinguished Professorship.

\section{References}

1. Jeffrey G A 1997 In An Introduction to Hydrogen Bonding (New York: Oxford University Press)

2. Arunan E, Desiraju G R, Klein R A, Sadlej J, Scheiner S, Alkorta I, Clary D C, Crabtree R H, Dannenberg J J, Hobza P, Kjaergaard H G, Legon A C, Mennucci B and Nesbitt D J 2011 Definition of the Hydrogen Bond Pure Appl. Chem. 831637

3. (a) Desiraju G R 2011 A bond by any other name Angew. Chem. Int. Ed. 50 52; (b) Desiraju G R 1991 The C-H...O Hydrogen Bond in Crystals: What is it? Acc. Chem. Res. 24290

4. Hobza P and Havlas Z 2000 Blue-Shifting Hydrogen Bonds Chem. Rev. 1004253

5. Joseph J and Jemmis E D 2007 Red-, Blue-, or No-Shift in Hydrogen Bonds: A Unified Explanation J. Am. Chem. Soc. 1294620

6. Li X, Liu L and Schlegel H B 2002 On the physical origin of blue-shifted hydrogen bonds J. Am. Chem. Soc. 124 9639

7. Alabugin I V, Monoharan M, Peabody S and Weinhold F 2003 Electronic basis of improper hydrogen bonding: a subtle balance of hyperconjugation and rehybridization J. Am. Chem. Soc. 1255973 
8. Li A Y 2007 Chemical origin of blue- and redshifted hydrogen bonds: intramolecular hyperconjugation and its coupling with intermolecular hyperconjugation $J$. Chem. Phys. 126154102

9. (a) Hobza P 2001 The H-index unambiguously discriminates between hydrogen bonding and improper blueshifting hydrogen bonding Chem. Phys. Phys. Chem. 3 2555; (b) Hobza P and Havlas Z 2002 Improper, blueshifting hydrogen bond Theor. Chem. Acc. 108325

10. Hobza P, Špirko V, Havlas Z, Buchhold K, Reimann B, Barth H and Brutschy B 1999 Anti-hydrogen bond between chloroform and fluorobenzene Chem. Phys. Lett. 299180

11. Rozenberg M, Loewenschuss A and Marcus Y 2000 An empirical correlation between stretching vibration redshift and hydrogen bond length Phys. Chem. Chem. Phys. 22699

12. Scheiner S and Kar T 2002 Red- versus Blue-Shifting Hydrogen Bonds: Are There Fundamental Distinctions J. Phys. Chem. A 1061784

13. Gilli G and Gilli P 2000 Towards an unified hydrogenbond theory J. Mol. Struct. 5521

14. Hermansson K 2002 Blue-Shifting Hydrogen Bonds $J$. Phys. Chem. A 1064695

15. Wang J, Feng Y, Liu L, Li X and Guo Q 2004 On the correlation between the blue shift of hydrogen bonding and the proton donor-proton acceptor distance Chin. J. Chem. 22642

16. Lu P, Liu G and Li J 2005 Existing problems in theoretical determination of red-shifted or blue-shifted hydrogen bond J. Mol. Struct. 72395

17. Wang S, Sahu P K and Lee S 2005 Intermolecular orbital repulsion effect on the blue-shifted hydrogen bond Chem. Phys. Lett. 406143

18. Hoja J, Sax, A F and Szalewicz K 2014 Is Electrostatics Sufficient to Describe Hydrogen-Bonding Interactions? Chem. Eur. J. 202292

19. Senthilkumar L, Ghanty T K, Ghosh S K and Kolandaivel P 2006 Hydrogen bonding in substituted formic acid dimers J. Phys. Chem. 11012623

20. Senthilkumar L, Ghanty T K and Ghosh S K 2006 Electron density and energy decomposition analysis in hydrogen-bonded complexes of azabenzenes with water, acetamide, and thioacetamide J. Phys. Chem. A 1097575
21. Kolandaivel P and Nirmala V 2004 Study of proper and improper hydrogen bonding using Bader's atoms in molecules (AIM) theory and NBO analysis J. Mol. Struct. 69433

22. Parthasarathy R, Subramanian V and Sathyamurthy N 2006 Hydrogen bonding without borders: An atom in molecules perspective J. Phys. Chem. Lett. 1103349

23. Dey A, Mondal S I, Sen S, Ghosh D and Patwari G N 2014 Electrostatics determine vibrational frequency shifts in hydrogen bonded complexes Phys. Chem. Chem. Phys. 1625247

24. Dey A, Mondal S I, Sen S and Patwari G N 2016 Spectroscopic and $\mathrm{Ab}$ Initio Investigation of $\mathrm{C}-\mathrm{H} \cdots \mathrm{N}$ Hydrogen-Bonded Complexes of Fluorophenylacetylenes: Frequency Shifts and Correlations ChemPhysChem 172509

25. Shirhatti P R, Maity D K and Wategaonkar S 2013 C $\mathrm{H}$... Y hydrogen bonds in the complexes of p-cresol and p-cyanophenol with fluoroform and chloroform J. Phys. Chem. A 1172307

26. Banerjee P, Mukhopadhyay D P and Chakraborty T 2015 On the origin of donor $\mathrm{O}-\mathrm{H}$ bond weakening in phenolwater complexes J. Chem. Phys. 143204306

27. Goswami M and Arunan E 2009 The hydrogen bond: a molecular beam microwave spectroscopist's view with a universal appeal Phys. Chem. Chem. Phys. 118974

28. Saggu M, Levinson N M and Boxer S G 2012 Experimental Quantification of Electrostatics in $\mathrm{X}-\mathrm{H} \cdots \pi$ Hydrogen Bonds J. Am. Chem. Soc. 13418986

29. Schmidt M W, Baldridge K K, Botaz J A, Elbert S T, Gordon M S, Jensen J, Koseki S, Matsunaga N, Nguyen K A, Su S, Windus T L, Dupuis M and Montgomery J A 1993 General atomic and molecular electronic structure system J. Comp. Chem. 141347

30. Gilli P, Bertolasi V, Ferretti V and Gilli G 1994 Covalent Nature of the Strong Homonuclear Hydrogen Bond. Study of the $\mathrm{O}-\mathrm{H}$...O System by Crystal Structure Correlation Methods J. Am. Chem. Soc. 116909

31. Umeyama H and Morokuma K 1977 The Origin of Hydrogen Bonding. An Energy Decomposition Study J. Am. Chem. Soc. 991316

32. Saha R, Pan S, Frenking G, Chattaraj P K and Merino G 2017 The strongest CO binding and the highest C-O stretching frequency Phys. Chem. Chem. Phys. 192286 